\title{
Probing invisible decay of a dark photon at BESIII and a future Super Tau Charm Factory via monophoton searches
}

\author{
Yu Zhang $\odot,{ }^{1,2, *}$ Wei-Tao Zhang $\odot,{ }^{1}$ Mao Song $\odot,{ }^{2}$ Xue-An Pan $\odot,{ }^{2}$ Zhong-Ming Niu, ${ }^{1,2}$ and Gang $\mathrm{Li}^{2}$ \\ ${ }^{1}$ Institutes of Physical Science and Information Technology, Anhui University, Hefei 230601, China \\ ${ }^{2}$ School of Physics and Materials Science, Anhui University, Hefei 230039,China
}

(Received 21 July 2019; published 6 December 2019)

\begin{abstract}
We propose probing sub-GeV dark photon decays into lighter dark matter using monophoton events at the BESIII detector and future Super Tau Charm Factory (STCF). We compute the cross section due to the dark photon associated a standard model photon production, and study the corresponding standard model irreducible/reducible backgrounds. By using the luminosity about $14 \mathrm{fb}^{-1}$ collected at the BESIII detector since 2012, we derive new expected leading limits of the mixing strength $\varepsilon, \varepsilon \lesssim(1.3-1.7) \times 10^{-4}$, in the mass range of $0.04 \mathrm{GeV} \lesssim m_{A^{\prime}} \lesssim 3 \mathrm{GeV}$. With the luminosity of $30 \mathrm{ab}^{-1}$, STCF running at $\sqrt{s}=2 \mathrm{GeV}$, can probe $\varepsilon$ down to $5.1 \times 10^{-6}$ when $m_{A^{\prime}}=1 \mathrm{GeV}$. For models of scalar and fermionic light thermal dark matter production via dark photon, we present the constrains on the dimensionless dark matter parameter $y=\varepsilon^{2} \alpha_{D}\left(m_{\chi} / m_{A^{\prime}}\right)^{4}$ as function of the DM mass $m_{\chi}$ at BESIII and future STCF, conventionally assuming the dark coupling constant $\alpha_{D}=0.5$ and $m_{A^{\prime}}=3 m_{\chi}$. We find that BESIII can exclude models of scalar, Majorana, and pseudo-Dirac (with a small splitting) DM for the mass region 0.04-1 GeV, 0.05-1 GeV and 0.4-1 GeV respectively. For values $\alpha_{D} \lesssim 0.005$, combining the results from $2 \mathrm{GeV}$ STCF with the luminosity of $30 \mathrm{ab}^{-1}$ and $B A B A R$, one can exclude the above three DM models in the mass region $0.001 \mathrm{GeV} \lesssim m_{\chi} \lesssim 1 \mathrm{GeV}$.
\end{abstract}

DOI: 10.1103/PhysRevD.100.115016

\section{INTRODUCTION}

To investigate the nature of dark matter (DM) particle is one of the most pressing issues in modern physics. So far, we have only been able to probe the DM through its gravitational effects with visible matter. It is widely postulated that DM interacts very weakly with ordinary matter, since terrestrial searches have not yielded any results yet. An exciting attempt is that, besides the gravity, one can introduce an extra $U_{D}(1)$ force carrier, also referred to as dark photon $A^{\prime}$. The dark photon can provide a natural scenario for DM interactions, which is neutral under the SM gauge symmetries, but couples to the SM photon via kinetic mixing [1-6]. The kinetic mixing term can be described as

$$
\mathcal{L}_{\text {kinetic mixing }}=-\frac{\varepsilon}{2} F_{\mu \nu}^{\prime} F^{\mu \nu}
$$

and parameterized by the mixing strength $\varepsilon \ll 1$. Here $F_{\mu \nu}^{\prime}=\partial_{\mu} A_{\nu}^{\prime}-\partial_{\nu} A_{\mu}^{\prime}$ is the field strength of $A^{\prime}$, resulting in the interaction

*dayu@ahu.edu.cn, dayu@nju.edu.cn

Published by the American Physical Society under the terms of the Creative Commons Attribution 4.0 International license. Further distribution of this work must maintain attribution to the author(s) and the published article's title, journal citation, and DOI. Funded by SCOAP ${ }^{3}$.

$$
\mathcal{L}_{\text {int }}=\varepsilon e A_{\mu}^{\prime} J_{e m}^{\mu}
$$

of dark photon with the electromagnetic current $J_{e m}^{\mu}$ with a strength $\varepsilon e$, where $e$ is the electromagnetic coupling. In order to explain observational astroparticle anomalies, the dark photon should be relatively light, with a mass in the $\mathrm{MeV}$ to $\mathrm{GeV}$ range [5]. Furthermore, a sub-GeV $A^{\prime}$ with $\varepsilon \simeq 10^{-3}$ can also explain the $3.6 \sigma$ deviation from the SM prediction of the muon anomalous magnetic moment $(g-2)_{\mu}[7,8]$.

The decay modes of the dark photon depend on its mass and couplings, as well as on the particle spectrum of the dark sector. Since there are no firm predictions for the dark photon, various experiments have been searched for it over a wide range of its mass and decay modes [9-11]. If the dark photon is lightest in the dark sector, its dominant decays are to the visible SM particles. The searches for such dark photon with the mass below a few $\mathrm{GeV}$ have been mainly performed in beam dump [12-23], fixed target [24-26], collider [27-32] and rare meson decay [33-43] experiments using narrow peak in the $e^{+} e^{-}$or $\mu^{+} \mu^{-}$ invariant mass spectra. If the lowest-mass DM state $\chi$ is sufficiently light, in particular $m_{\chi}<m_{A^{\prime}} / 2$, the $A^{\prime}$ would predominantly decay invisibly into the DM particles provided that $e_{D}>\varepsilon e . e_{D}$ is the coupling constant of the $U_{D}(1)$ gauge interactions. There are limits on invisible 
decays of the dark photon from kaon decays by the E787 [44] and E949 [45] experiments, $\pi^{0}$ decays by NA62 [46] experiment, searches for missing energy events in electronnucleus scattering by NA64 [47-49] experiment, and monophoton searches by BABAR [50].

Over the past few years, there has been substantial effort to probe the dark photon. In the Ref. [51], they develop a framework for recasting dark photon searches to obtain constraints on more general theories. For the invisible decay of the dark photon, the constraints from existing data and proposed searches are given in Refs. [44-57]. In this paper, we focus on the search for the invisible decay of the dark photon at the BESIII detector and future Super Tau Charm Factory (STCF). The BESIII detector is operated at the Beijing Electron Positron Collider (BEPCII), which is a double ring $e^{+} e^{-}$collider running at the center-ofmass (c.m.) energy $\sqrt{s}$ from 2.0 to $4.6 \mathrm{GeV}$ with a peaking luminosity of $10^{33} \mathrm{~cm}^{-2} \mathrm{~s}^{-1}$. The STCF is a projected electron-positron collider operating in the range of centerof-mass energies from 2.0 to $7.0 \mathrm{GeV}$ with the peak luminosity of about $10^{35} \mathrm{~cm}^{-2} \mathrm{~s}^{-1}[58,59]$. We assume that the decay width of the $A^{\prime}$ is negligible compared to the experimental resolution, and the invisible branching ratio $\operatorname{Br}\left(A^{\prime} \rightarrow \chi \bar{\chi}\right) \simeq 100 \%$. The cleanest collider signature of such particles is the process $e^{+} e^{-} \rightarrow \gamma A^{\prime}$, followed by invisible decay of the $A^{\prime}$, which is monochromatic single photon production accompanied by significant missing energy and momentum. The monophoton signal has been investigated at BESIII detector [60-63]. Here we use the monophoton signature to probe invisible decay of dark photon at the BESIII detector and STCF.

The rest of the paper is organized as follows. In Sec. II, we study the monophoton signature arising from dark photon production and from the SM backgrounds. The results on the sensitivity for invisible decay of dark photon at BESIII and future STCF are presented in Sec. III. The constraints on light thermal dark matter are reported in Sec. IV. We summarize our findings in Sec. V.

\section{SIGNALS AND BACKGROUNDS}

At the electron colliders, the dark photon can be investigated in the process $e^{+} e^{-} \rightarrow \gamma A^{\prime}$, whose diagrams are shown in Fig. 1, with its subsequent decay to lighter DM.

In this paper, we only consider the leading order contributions for the signal and background. The differential cross section for an on-shell $A^{\prime}$ and a photon production process $e^{+} e^{-} \rightarrow \gamma A^{\prime}$ is [64]

$$
\frac{d \sigma_{\gamma A^{\prime}}}{d z_{\gamma}}=\frac{2 \pi \varepsilon^{2} \alpha^{2}}{s}\left(1-\frac{m_{A^{\prime}}^{2}}{s}\right) \frac{1+z_{\gamma}^{2}+\frac{4 s m_{A^{\prime}}^{2}}{\left(s-m_{A^{\prime}}^{2}\right)^{2}},}{\left(1+z_{\gamma}\right)\left(1-z_{\gamma}\right)},
$$

where $\alpha$ is the fine structure constant, $z_{\gamma} \equiv \cos \theta_{\gamma}$ with $\theta_{\gamma}$ being the relative angle between the electron beam axis and

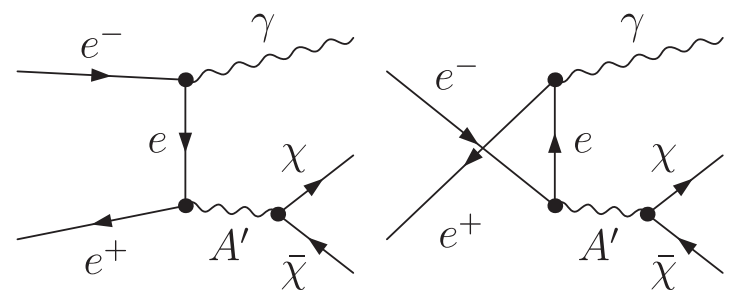

FIG. 1. The Feynman diagrams for the production of an onshell $A^{\prime}$ and a photon, in which we assume the $A^{\prime}$ subsequently decays to lighter dark matter.

the photon momentum, $s$ is the square of the center-of-mass energy, $m_{A^{\prime}}$ is the mass of the dark photon. The photon energy $E_{\gamma}$ in the center-of-mass frame is related to the dark photon mass as

$$
E_{\gamma}=\frac{s-m_{A^{\prime}}^{2}}{2 \sqrt{s}}
$$

The cross section after integrating the polar angle $\theta_{\gamma}$ is given as [64]

$$
\begin{aligned}
\sigma_{\gamma A^{\prime}}= & \frac{2 \pi \varepsilon^{2} \alpha^{2}}{s}\left(1-\frac{m_{A^{\prime}}^{2}}{s}\right) \\
& \times\left[\left(1+\frac{2 s m_{A^{\prime}}^{2}}{\left(s-m_{A^{\prime}}^{2}\right)^{2}}\right) \mathcal{Z}-z_{\gamma}^{\max }+z_{\gamma}^{\min }\right],
\end{aligned}
$$

where

$$
\mathcal{Z}=\ln \frac{\left(1+z_{\gamma}^{\max }\right)\left(1-z_{\gamma}^{\min }\right)}{\left(1-z_{\gamma}^{\max }\right)\left(1+z_{\gamma}^{\min }\right)}
$$

The irreducible SM backgrounds to the monophoton signature at electron-positron colliders are the $e^{+} e^{-} \rightarrow$ $\nu_{\ell} \bar{\nu}_{\ell} \gamma$ processes, where $\nu_{\ell}=\nu_{e}, \nu_{\mu}, \nu_{\tau}$ are the three standard model neutrinos. The corresponding Feynman diagrams are displayed in Fig. 2. For electron neutrinos, both $Z$-boson and $W$-boson diagrams contribute; for the muon and tau neutrinos only $Z$-boson diagrams. For the electronpositron colliders running with $\mathrm{GeV}$ beam energy, the diagram mediated by two $W$ bosons can be safely eliminated in our analysis, since it is suppressed by an additional $W$-boson propagator in comparison with other single

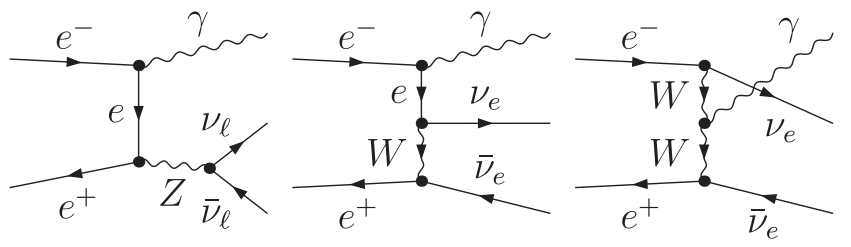

FIG. 2. The Feynman diagrams for the SM processes $e^{+} e^{-} \rightarrow \nu_{\ell} \bar{\nu}_{\ell} \gamma$, where $\nu_{\ell}=\nu_{e}, \nu_{\mu}, \nu_{\tau}$ are the three standard model neutrinos. 

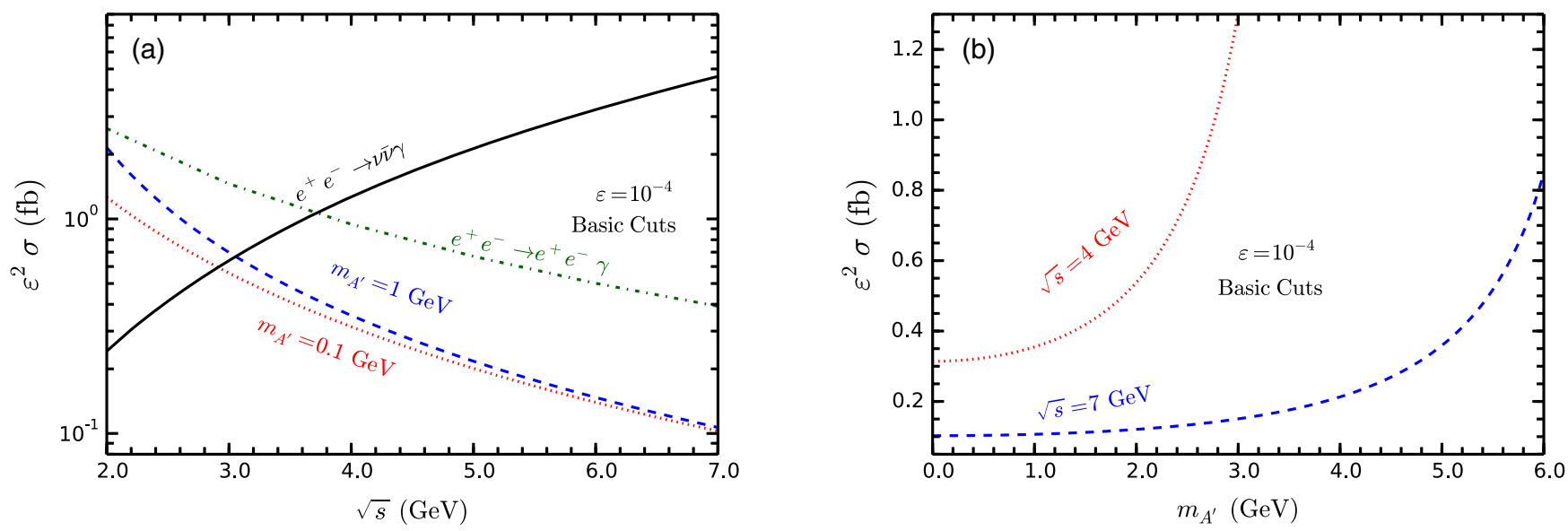

FIG. 3. (a) The total cross sections as a function of the collider energy for the irreducible SM background $e^{+} e^{-} \rightarrow \nu \bar{\nu} \gamma, \# 1$ reducible SM background $e^{+} e^{-} \rightarrow e^{+} e^{-} \gamma$, and for the production of an on-shell dark photon and a photon with $m_{A^{\prime}}=0.1 \mathrm{GeV}$ and $m_{A^{\prime}}=1 \mathrm{GeV}$, respectively. (b) The total cross sections as a function of the mass of the dark photon for the production of an on-shell dark photon and a photon with $\sqrt{s}=4 \mathrm{GeV}$ and $\sqrt{s}=7 \mathrm{GeV}$, respectively. All the results are obtained by adopting the "basic cuts" and setting $\varepsilon=10^{-4}$. For the reducible SM background $e^{+} e^{-} \rightarrow e^{+} e^{-} \gamma$, the cuts $\left|z_{e^{ \pm}}\right|>0.95$ are applied on the final electron and positron, and the cross section is multiplied by a factor of $10^{-8}$ to compare more intuitively.

$W$ or $Z$ mediator diagrams. The differential production cross section for the $e^{+} e^{-} \rightarrow \nu \bar{\nu} \gamma$ processes mediated by a single $W / Z$ boson is given by $[65,66]$

$\frac{d \sigma}{d E_{\gamma} d z_{\gamma}}=\frac{\alpha G_{F}^{2} s_{\gamma}^{2}}{4 \pi^{2} s E_{\gamma}\left(1-z_{\gamma}^{2}\right)} f\left(\sin \theta_{W}\right)\left[1+\frac{E_{\gamma}^{2}}{s_{\gamma}}\left(1+z_{\gamma}^{2}\right)\right]$

where $G_{F}$ is the Fermi constant, $f\left(\sin \theta_{W}\right)=8 \sin ^{4} \theta_{W}-$ $4 \sin ^{2} \theta_{W} / 3+1$ with $\theta_{W}$ being the weak mixing angle. Here we have integrated over the momenta of the final state neutrinos and summed all three neutrino flavors.

In Fig. 3(a), we present the total cross section as a function of the collider energy for the irreducible SM background $e^{+} e^{-} \rightarrow \nu \bar{\nu} \gamma$, as well as the production of an on-shell dark photon and a photon with $m_{A^{\prime}}=0.1 \mathrm{GeV}$ and $m_{A^{\prime}}=1 \mathrm{GeV}$, respectively. For the final photon, we adopt the cuts: $E_{\gamma}>25 \mathrm{MeV}$ in the barrel $\left(\left|z_{\gamma}\right|<0.8\right)$ or $E_{\gamma}>50 \mathrm{MeV}$ in the end-caps $\left(0.86<\left|z_{\gamma}\right|<0.92\right)$, following the cuts used by the BESIII Collaboration [67], which are defined as the "basic cuts" hereafter. We can see that the production rates for dark photon associated with one SM photon drop rapidly when the colliding energy increases; however, the monophoton cross section due to the SM irreducible processes grows with the colliding energy. Thus, electron collider with smaller colliding energy has a better sensitivity to search the invisible decay of dark photon when kinematics is accessible. In Fig. 3(b), we also provide the dependence of the total cross section for dark photon production on its mass when $\sqrt{s}=4 \mathrm{GeV}$ and $7 \mathrm{GeV}$ with the basic cuts. It can be seen that the production rates keep growing with the increment of the mass of dark photon.
Due to the limited detection capability of the subdetectors, the reducible backgrounds become important and should be investigated carefully. The reducible SM backgrounds mainly come from the $e^{+} e^{-} \rightarrow \gamma+\not X$ processes, where only one final state photon can be detected in the detectors, and $X$ denotes that the other particles are undetected because of the limitations of the detectors. The dominate reducible backgrounds include the processes $e^{+} e^{-} \rightarrow f \bar{f} \gamma$ and $e^{+} e^{-} \rightarrow \gamma \gamma \gamma,{ }^{1}$ which can be quite large with the final $f \bar{f}$ and $\gamma \gamma$ emitting in the solid angle region that is uncovered by detectors. Especially, for the radiative Bhabha scattering process $e^{+} e^{-} \rightarrow e^{+} e^{-} \gamma$, when both final state electron and positron go along the beam directions, the collinear singularity will arise in the $t$ channel diagrams, and cause large cross section $[62,68,69]$. We present the cross section for the reducible background $e^{+} e^{-} \rightarrow e^{+} e^{-} \gamma$ as a function of the collider energy in Fig. 3(a), with the cuts $\left|z_{e^{ \pm}}\right|>0.95$ on the final $e^{ \pm}$and the basic cuts for final photon, which is numerically evaluated with FEYNARTS [70] and FORMCALC [71] package. To compare more intuitively, the cross section is multiplied an factor of $10^{-8}$. One can see that, the contribution from the reducible background is super large, if only basic cuts are applied on the final photon. At the BESIII, photon reconstruction efficiencies are all more than $99 \%$ [72], we assume them to be $100 \%$ in our paper.

Due to momentum conservation in the transverse direction and energy conservation, the monophoton reducible background at the electron-positron colliders can be

\footnotetext{
${ }^{1}$ The reducible background from the $e^{+} e^{-} \rightarrow \gamma \gamma$ process vanishes because the BESIII and STCF detectors are arranged in a symmetric manner.
} 

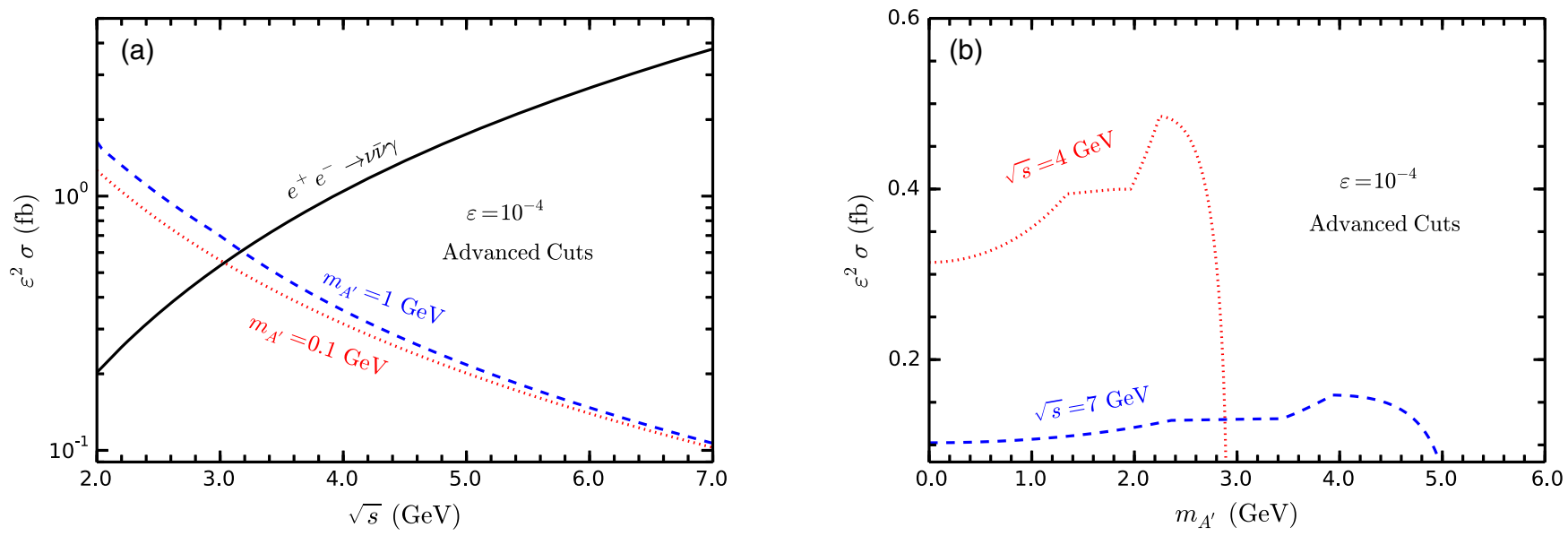

FIG. 4. Same as Fig. 3. All the results are obtained by adopting the advanced cuts and setting $\varepsilon=10^{-4}$.

removed by applying the detector cut [73] (Please see the details in the Appendix):

$$
E_{\gamma}>E_{b}\left(\theta_{\gamma}\right)=\frac{\sqrt{s}}{\left(1+\sin \theta_{\gamma} / \sin \theta_{b}\right)},
$$

on the final state photon, where the energy cut $E_{b}$ is the function of the polar angle $\theta_{b}$, and $\theta_{b}$ denotes the angle at the boundary of the subdetectors. We will collectively refer to the "basic cuts" and cut (8) as the "advanced cuts" hereafter. At the BESIII, we follow Ref. [62], and define the polar angel $\left|\cos \theta_{b}\right|=0.95$ after considering all the boundary of the subdetectors. When $\theta_{\gamma}=\pi / 2$, the energy cut $E_{b}$ achieves its minimum value $E_{b}^{\min } \simeq 0.24 \sqrt{s}$. In order to probe the sensitivity of STCF to dark photon, we assume that the sub-detectors of STCF have the same acceptance with the BESIII.

In Fig. 4, we present the same results with Fig. 3 by using the advanced cuts for the final photon. It is noted that the reducible SM backgrounds are removed by the advanced cuts. We can see that the production rates for dark photon and irreducible background in Fig. 4(a) have the same trend with Fig. 3(a) when the colliding energy increases. While the curves in Fig. 4(b) have different shapes with Fig. 3(b). As $m_{A^{\prime}}$ increases, the dark photon production rates starts to go up, reaches its maximum when $m_{A^{\prime}} \simeq 2.3(3.9) \mathrm{GeV}$ for $\sqrt{s}=4$ (7) GeV, and then quickly goes down.

To simulate the detector effects on the final state particles, we smear the energy for the final state photon using Gaussian distributions which take into account the energy resolution of the EMC at the BESIII detector as [74]

$$
\sigma(E) / E=2.3 \% / \sqrt{E / \mathrm{GeV}} \oplus 1 \%
$$

For the EMC at STCF, we assume the same energy resolution with BESIII to present a preliminary projection limit, because of the similarity of the two experiments.

\section{RESULTS AND CALCULATION OF THE LIMIT}

A large number of data have been accumulated by the BESIII detector at various running energies. We summarize the luminosities collected at BESIII in Table I since 2012 when the monophoton trigger was implemented [75]. The table is arranged by the center-of-mass $\sqrt{s}$, of which the taking year is also listed [76]. To probe millicharge for each BESIII colliding energy, we define $\chi_{i}^{2}(\varepsilon) \equiv S_{i}^{2} /\left(S_{i}+B_{i}\right)$ [61], where $S_{i}\left(B_{i}\right)$ is the number of signal (background) events, and the $95 \%$ confidence level (C.L.) upper bound on the millicharge, $\varepsilon_{95}^{i}$, is obtained by solving $\chi_{i}^{2}\left(\varepsilon_{95}^{i}\right)-$ $\chi_{i}^{2}(0)=2.71$. The expected $95 \%$ C.L. upper bound on millicharge from BESIII is obtained by demanding $\chi_{\mathrm{tot}}^{2}\left(\varepsilon_{95}\right)-\chi_{\mathrm{tot}}^{2}(0)=2.71$, where $\chi_{\mathrm{tot}}^{2}$ is the total chi-square, defined as $\chi_{\mathrm{tot}}^{2}(\varepsilon)=\sum_{i} \chi_{i}^{2}(\varepsilon)$.

TABLE I. The center-of-mass energy and corresponding luminosities collected from 2012 to 2018 at the BESIII detector. The 95\% C.L. upper limits on $\varepsilon$ for the $m_{A^{\prime}}=1.5 \mathrm{GeV}$ are listed in the last column. The last row shows the result combining all luminosities between 2012 and 2018.

\begin{tabular}{lccc}
\hline \hline Year & $\sqrt{s}(\mathrm{GeV})$ & $\mathcal{L}\left(\mathrm{fb}^{-1}\right)$ & $\varepsilon_{95}\left(\times 10^{-4}\right)$ \\
\hline 2015 & 2.125 & 0.1 & 5.3 \\
$2012+2018$ & 3.097 & 1.8 & 1.7 \\
2017 & 3.515 & 0.46 & 3.6 \\
2018 & 3.554 & 0.13 & 6.5 \\
$2012+2018$ & 3.686 & 1.0 & 2.7 \\
2017 & 3.872 & 0.22 & 5.6 \\
2016 & 4.18 & 3.1 & 2.1 \\
2013 & 4.23 & 1.05 & 3.1 \\
2013 & 4.26 & 0.83 & 3.5 \\
2017 & 4.28 & 3.8 & 2.0 \\
2012 & 4.36 & 0.5 & 4.4 \\
2014 & 4.42 & 1 & 3.4 \\
2014 & 4.6 & 0.5 & 4.7 \\
$12-18$ & - & 14.49 & 1.1 \\
\hline \hline
\end{tabular}




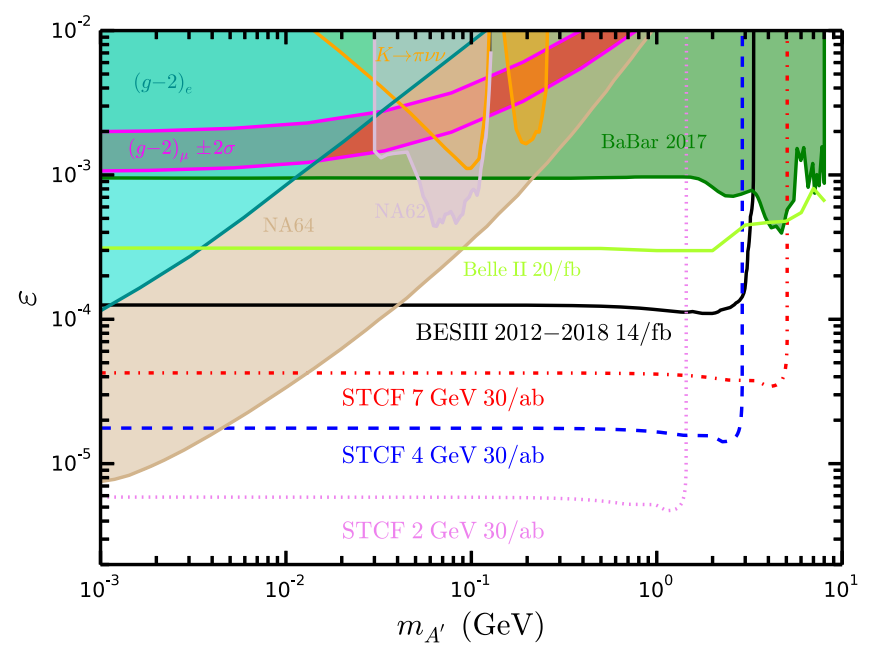

FIG. 5. The expected 95\% C.L. exclusion limits on dark photon $A^{\prime}$ mixing strength $\varepsilon$ as a function of the mass $m_{A^{\prime}}$ at BESIII using the luminosities collected during 2012-2018 (solid black). The STCF sensitivity curves are obtained assuming 30/ab integrated luminosity data being collected at $\sqrt{s}=2 \mathrm{GeV}$ (dotted magenta), $\sqrt{s}=4 \mathrm{GeV}$ (dashed blue), $\sqrt{s}=7 \mathrm{GeV}$ (dot-dashed red), respectively. The shaded regions show the existing bounds on $\varepsilon$ in channels where $A^{\prime}$ is allowed to decay invisibly from the $B A B A R$ [50], NA64 [49], NA62 [46] experiments, and the measurement for $\mathrm{BR}\left(K^{+} \rightarrow \pi^{+} \nu \bar{\nu}\right)$ by the E787 [44] and E949 [45] experiments, as well as the anomalous muon magnetic moment $(g-2) \mu$ favored area [8]. The projected upper limits on $\varepsilon$ for the process $e^{+} e^{-} \rightarrow \gamma A^{\prime}\left(\rightarrow\right.$ invisible), for a $20 \mathrm{fb}^{-1}$ Belle II data set (solid green) [77] are also given.

In the last column of Table I, we list $\varepsilon_{95}$ for the $m_{A^{\prime}}=$ $1.5 \mathrm{GeV}$ at each running energy at BESIII. The last row shows the limit combining all luminosities between 2012 and 2018. We can see that, when $m_{A^{\prime}}=1.5 \mathrm{GeV}$, the expected upper bound on dark photon strength $\varepsilon$ at the $95 \%$ C.L. can reach about $1.1 \times 10^{-4}$.

In Fig. 5, we show the expected 95\% C.L. exclusion upper limits on $\varepsilon$ as a function of the mass $m_{A^{\prime}}$ via monophoton searches by using the luminosities presented in Table I at BESIII (solid black). We also present the STCF sensitivity on $\varepsilon$ simply assuming about $30 \mathrm{ab}^{-1}$ data collected at $\sqrt{s}=2 \mathrm{GeV}$ (dotted magenta), $\sqrt{s}=4 \mathrm{GeV}$ (dashed blue), $\sqrt{s}=7 \mathrm{GeV}$ (dot-dashed red), respectively. The preexisting experimental constraints are also shown, which include the bounds in channels where $A^{\prime}$ is allowed to decay invisibly from the NA62 [46], NA64 [49], $B A B A R$ [50], the measurement for $\operatorname{BR}\left(K^{+} \rightarrow \pi^{+} \nu \bar{\nu}\right)$ by the E787 [44] and E949 [45] experiments, as well as the anomalous muon magnetic moment $(g-2) \mu$ favored area [8]. The projected upper limits on $\varepsilon$ for the process $e^{+} e^{-} \rightarrow \gamma A^{\prime}\left(\rightarrow\right.$ invisible), for a $20 \mathrm{fb}^{-1}$ Belle II data set (solid green) [77] are also given. We can see that BESIII with the collected luminosity of about $14 \mathrm{fb}^{-1}$ can provide new leading upper limits to the mixing strength $\varepsilon$ of the dark photon in the mass range $0.04 \mathrm{GeV} \lesssim m_{A^{\prime}} \lesssim 3 \mathrm{GeV}$, of which the sensitivity is significantly better than future Belle II experiments with $20 \mathrm{fb}^{-1}$. When $m_{A}^{\prime}=1 \mathrm{GeV}$, the limit of $\varepsilon$ can be probed by BESIII down to $1.1 \times 10^{-4}$, which outperform the result from BABAR [50] about one order. From the sensitivity on $\varepsilon$ at STCF with different collider energies, we can see that the lower collider energy has better sensitivity than the higher energy, in spite of it touches smaller mass range. For example, with the luminosity of $30 \mathrm{ab}^{-1}, 2 \mathrm{GeV}$ STCF can probe $\varepsilon$ down to $5.1 \times$ $10^{-6}$ when $m_{A}^{\prime}=1 \mathrm{GeV}$, which outmatches $7 \mathrm{GeV}$ STCF about 7 times.

\section{CONSTRAINTS ON LIGHT THERMAL DARK MATTER}

In this section, we extend our discussions for the constraints on light thermal dark matter (LTDM). The existence of thermal DM is arguably one of the most compelling possibilities, and has driven much of DM experiments over the past several years. Among the thermal DM parameter space, the LTDM annihilating directly into SM particles (the "thermal relic target") sticks out for its predictiveness and testability [11]. In the left panel of Fig. 6, we plot the expected 95\% C.L. values on the dimensionless DM annihilation cross section parameter $y=\varepsilon^{2} \alpha_{D}\left(m_{\chi} / m_{A^{\prime}}\right)^{4}$ as function of the DM mass $m_{\chi}$ at BESIII and future STCF, where $\alpha_{D}=e_{D}^{2} / 4 \pi$, under the conventional assumption $m_{A^{\prime}}=3 m_{\chi}$ and $\alpha_{D}=0.5$, and compare them with different experimental exclusion regions. The favored parameters for scalar, pseudo-Dirac (with a small splitting) and Majorana scenario of LTDM into account the observed relic DM density [48] are also shown. We can see that the direct search for the dark photon invisible decay at BESIII via monophoton searches excludes model of scalar, Majorana, and pseudo-Dirac (with a small splitting) DM for the mass region 0.04$1 \mathrm{GeV}, 0.05-1 \mathrm{GeV}$, and $0.4-1 \mathrm{GeV}$, respectively. The choice of $\alpha_{D}=0.5$ is compatible with the bounds in Ref. [78] based on the running of the dark gauge coupling. However, it is important to note that the DM signal yields in our analyses are primarily sensitive to $\varepsilon^{2}$, same as other accelerator experiments, such as NA64 [48,49], different from $\varepsilon^{4} \alpha_{D}$ at the beam dump experiments, such as LSND [79,80], E137 [81], MiniBooNE [82]. Therefore, our limits will be much stronger for sufficiently small values of $\alpha_{D}$. In the right panel of Fig. 6, we present all the limits and bounds with $m_{A^{\prime}}=3 m_{\chi}$ and $\alpha_{D}=0.005$. We can see that, for this or smaller values of $\alpha_{D}$, the model of scalar and Majorana DM production via dark photon can be excluded by combining the NA64 [49] and BABAR [50] limits. Further combined with the limits from $2 \mathrm{GeV}$ STCF with $30 \mathrm{ab}^{-1}$ data and BABAR [50], the model of pseudo-Dirac (with a small splitting) DM can also be excluded in the entire plotted mass region. 

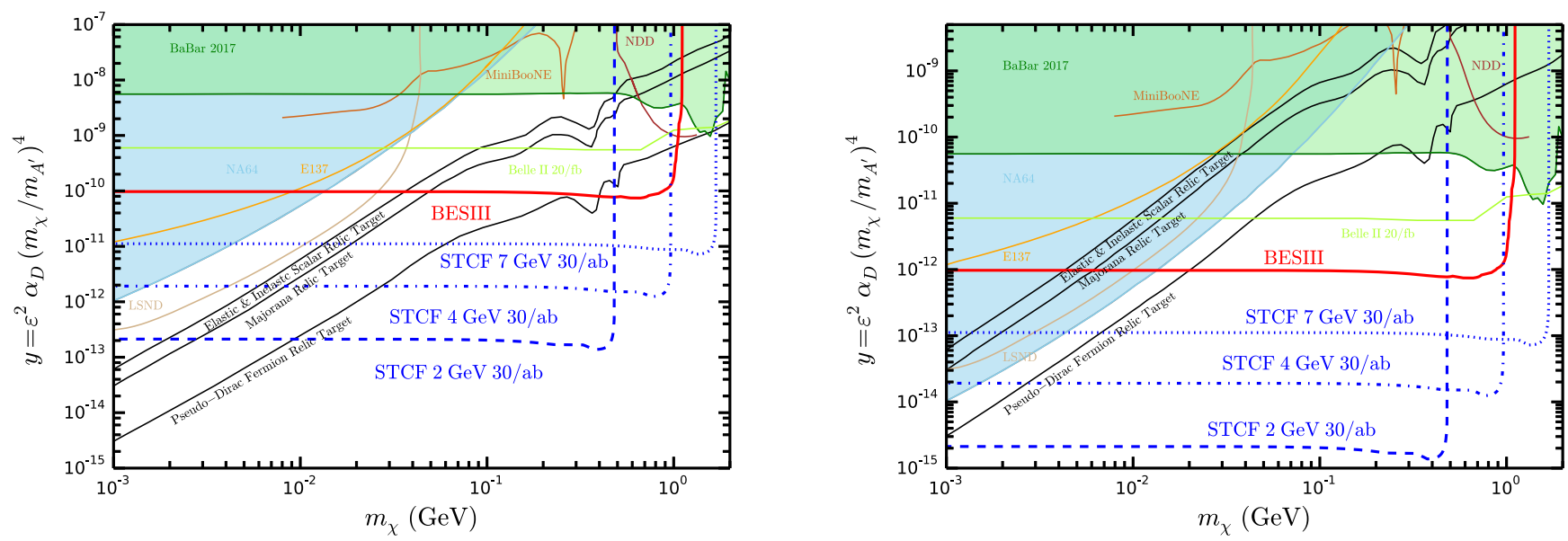

FIG. 6. The expected $95 \%$ C.L. exclusion limits on the parameter $y$ as a function of the mass $m_{\chi}$ from BESIII using the data collected during 2012-2018, as well as the future STCF. The STCF sensitivity curves are obtained assuming 30/ab integrated luminosity data being collected at $\sqrt{s}=2,4,7 \mathrm{GeV}$. The limits in the left panel are calculated under the conventional assumption $m_{A^{\prime}}=3 m_{\chi}$ and $\alpha_{D}=0.5$. In the right panel, the limits are shown for $\alpha_{D}=0.005$. The existing limits are obtained in Refs. [10,48,49,83-85] from the results of the NA64 [49], LSND [79,80], E137 [81], BABAR [50], MiniBooNE [82], and nucleon direct detection (NDD) [86] experiments based on the missing mass, missing energy, and missing momentum approaches. The favored parameters for the scalar, Majorana, and pseudo-Dirac of LTDM to account for the observed relic DM density are shown as the solid lines [48].

\section{SUMMARY}

In this work, we propose probing the invisible decay of dark photon via the monophoton signature at the BESIII detector and future STCF. The dark photon mixes with the SM photon and decays dominantly invisible into light DM particles $A^{\prime} \rightarrow \chi \bar{\chi}$. New leading constraints on the mixing strength $\varepsilon$ can be obtained in the mass range $0.04 \mathrm{GeV} \lesssim$ $m_{A^{\prime}} \lesssim 3 \mathrm{GeV}$ by using the current BESIII luminosities. We also present the sensitivity on $\varepsilon$ at future STCF with $\sqrt{s}=2$, 4, $7 \mathrm{GeV}$ assuming about $30 \mathrm{ab}^{-1}$ luminosity. In addition, we discuss the constraints on light termal dark matter. Using conventional choices, we provide the expected 95\% C.L. limits on the dimensionless DM annihilation cross section parameter $y$. We find that the BESIII results can be expected to expand the search for DM to $y$ values about two orders of magnitude smaller than $B A B A R$ [50]. For values $\alpha_{D}=0.005$ or smaller, the models for scalar and Majorana DM production via dark photon portal can be excluded by the combined results from direct searches of $A^{\prime}$ invisible decay in NA64 [49] and BABAR [50] experiments; the model for pseudo-Dirac (with a small splitting) can also be excluded by the combined results from $2 \mathrm{GeV}$ STCF with $30 \mathrm{ab}^{-1}$ luminosity and $B A B A R$ [50] for the mass region $0.001 \mathrm{GeV} \lesssim m_{\chi} \lesssim 1 \mathrm{GeV}$. In this paper, we mainly focus on the expected limits BESIII and future STCF can probe and provide referential suggestions for experiments.

\section{ACKNOWLEDGMENTS}

We thank Zuowei Liu, Dayong Wang, Lei Zhang, and Xiaorong Zhou for helpful discussions. This work was supported in part by the National Natural Science Foundation of China (Grants No. 11805001, No. 11305001,
No. 11575002, No. 11675033, No. 11747317 and No. 11875070) and the Key Research Foundation of the Education Ministry of Anhui Province of China (Grant No. KJ2017A032).

\section{APPENDIX: THE DETAILS TO REMOVE THE REDUCIBLE BACKGROUNDS}

Here, we take the most annoying reducible background $e^{+} e^{-} \rightarrow e^{+} e^{-} \gamma$ for example. At leading order, the conservation of transverse momentum and energy in the centerof-mass frame gives rise to

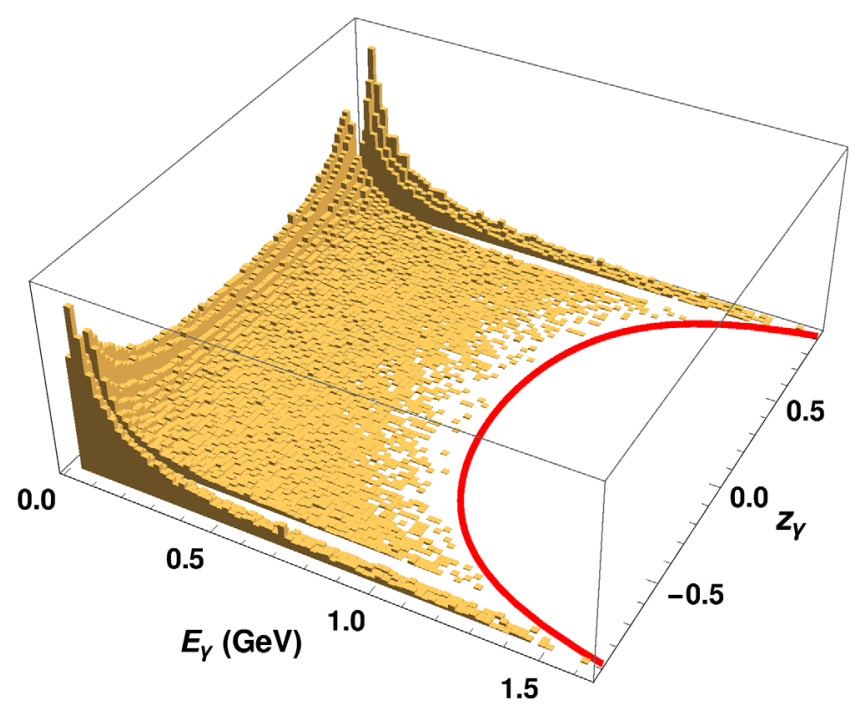

FIG. 7. Photon $E_{\gamma}-z_{\gamma}$ distribution in $e^{+} e^{-} \rightarrow e^{+} e^{-} \gamma$ with $\sqrt{s}=4 \mathrm{GeV}$ and $\left|\cos \theta_{e^{ \pm}}\right| \geq 0.95$. The red curve indicates $E_{\gamma}=\sqrt{s} /\left(1+\sin \theta_{\gamma} / \sin \theta_{b}\right)$. 


$$
\begin{gathered}
E_{\gamma} \sin \theta_{\gamma}-E_{1} \sin \theta_{1}-E_{2} \sin \theta_{2}=0 \\
E_{\gamma}+E_{1}+E_{2}=\sqrt{s},
\end{gathered}
$$

where $E_{\gamma}, E_{1}$, and $E_{2}$ are the final photon and $e^{ \pm}$energies, and $\theta_{\gamma}, \theta_{1}$, and $\theta_{2}$ are their polar angles. Since the final $e^{ \pm}$ escape from the uncovered region of the detectors, one needs

$$
\left|\cos \theta_{1}\right| \geq\left|\cos \theta_{b}\right| \&\left|\cos \theta_{2}\right| \geq\left|\cos \theta_{b}\right| \text {. }
$$

From above relations, we find that when both final state $e^{ \pm}$are emitted at the boundary of the EMC, i.e., $\sin \theta_{1}=\sin \theta_{2}=\sin \theta_{b}$, the maximum energy of the monophoton occurs for certain polar angle

$$
E_{b}\left(\theta_{\gamma}\right)=\frac{\sqrt{s}}{\left(1+\sin \theta_{\gamma} / \sin \theta_{b}\right)} .
$$

In Fig. 7, we present the final photon $E_{\gamma}-z_{\gamma}$ distribution using Monte-Carlo simulation with $10^{6}$ events for the process $e^{+} e^{-} \rightarrow e^{+} e^{-} \gamma$ at leading order with $\sqrt{s}=4 \mathrm{GeV}$ and $\left|\cos \theta_{e^{ \pm}}\right| \geq 0.95$. The basic cuts are applied to the final photon. The red curve indicates $E_{\gamma}=\sqrt{s} /$ $\left(1+\sin \theta_{\gamma} / \sin \theta_{b}\right)$. We can see that the above cut is efficient to remove the reducible background.
[1] L. B. Okun, Zh. Eksp. Teor. Fiz. 83, 892 (1982) [Sov. Phys. JETP 56, 502 (1982)].

[2] P. Galison and A. Manohar, Phys. Lett. 136B, 279 (1984).

[3] B. Holdom, Phys. Lett. 166B, 196 (1986).

[4] M. Pospelov, A. Ritz, and M. B. Voloshin, Phys. Lett. B 662, 53 (2008).

[5] N. Arkani-Hamed, D. P. Finkbeiner, T. R. Slatyer, and N. Weiner, Phys. Rev. D 79, 015014 (2009).

[6] J. D. Bjorken, R. Essig, P. Schuster, and N. Toro, Phys. Rev. D 80, 075018 (2009).

[7] M. Pospelov, Phys. Rev. D 80, 095002 (2009).

[8] G. W. Bennett et al. (Muon g-2 Collaboration), Phys. Rev. D 73, 072003 (2006).

[9] R. Essig et al., arXiv:1311.0029.

[10] J. Alexander et al., arXiv:1608.08632.

[11] M. Battaglieri et al., arXiv:1707.04591.

[12] F. Bergsma et al. (CHARM Collaboration), Phys. Lett. 166B, 473 (1986).

[13] A. Konaka et al., Phys. Rev. Lett. 57, 659 (1986).

[14] E. M. Riordan et al., Phys. Rev. Lett. 59, 755 (1987).

[15] J. D. Bjorken, S. Ecklund, W. R. Nelson, A. Abashian, C. Church, B. Lu, L. W. Mo, T. A. Nunamaker, and P. Rassmann, Phys. Rev. D 38, 3375 (1988).

[16] A. Bross, M. Crisler, S. H. Pordes, J. Volk, S. Errede, and J. Wrbanek, Phys. Rev. Lett. 67, 2942 (1991).

[17] M. Davier and H. Nguyen Ngoc, Phys. Lett. B 229, 150 (1989).

[18] C. Athanassopoulos et al. (LSND Collaboration), Phys. Rev. C 58, 2489 (1998).

[19] P. Astier et al. (NOMAD Collaboration), Phys. Lett. B 506, 27 (2001).

[20] J. Blumlein and J. Brunner, Phys. Lett. B 701, 155 (2011).

[21] S. Andreas, C. Niebuhr, and A. Ringwald, Phys. Rev. D 86, 095019 (2012).

[22] J. Blmlein and J. Brunner, Phys. Lett. B 731, 320 (2014).

[23] D. Banerjee et al. (NA64 Collaboration), Phys. Rev. Lett. 120, 231802 (2018).

[24] H. Merkel et al. (A1 Collaboration), Phys. Rev. Lett. 106, 251802 (2011).
[25] S. Abrahamyan et al. (APEX Collaboration), Phys. Rev. Lett. 107, 191804 (2011).

[26] H. Merkel et al., Phys. Rev. Lett. 112, 221802 (2014).

[27] B. Aubert et al. (BABAR Collaboration), Phys. Rev. Lett. 103, 081803 (2009).

[28] D. Curtin et al., Phys. Rev. D 90, 075004 (2014).

[29] J. P. Lees et al. (BABAR Collaboration), Phys. Rev. Lett. 113, 201801 (2014).

[30] A. Anastasi et al., Phys. Lett. B 750, 633 (2015).

[31] M. Ablikim et al. (BESIII Collaboration), Phys. Lett. B 774, 252 (2017).

[32] R. Aaij et al. (LHCb Collaboration), Phys. Rev. Lett. 120, 061801 (2018).

[33] G. Bernardi et al., Phys. Lett. 166B, 479 (1986).

[34] R. M. Drees et al. (SINDRUM I Collaboration), Phys. Rev. Lett. 68, 3845 (1992).

[35] F. Archilli et al. (KLOE-2 Collaboration), Phys. Lett. B 706, 251 (2012).

[36] S. N. Gninenko, Phys. Rev. D 85, 055027 (2012).

[37] D. Babusci et al. (KLOE-2 Collaboration), Phys. Lett. B 720, 111 (2013).

[38] P. Adlarson et al. (WASA-at-COSY Collaboration), Phys. Lett. B 726, 187 (2013).

[39] G. Agakishiev et al. (HADES Collaboration), Phys. Lett. B 731, 265 (2014).

[40] D. Babusci et al. (KLOE-2 Collaboration), Phys. Lett. B 736, 459 (2014).

[41] A. Adare et al. (PHENIX Collaboration), Phys. Rev. C 91, 031901 (2015).

[42] J. R. Batley et al. (NA48/2 Collaboration), Phys. Lett. B 746, 178 (2015).

[43] A. Anastasi et al. (KLOE-2 Collaboration), Phys. Lett. B 757, 356 (2016).

[44] S. Adler et al. (E787 Collaboration), Phys. Rev. Lett. 88, 041803 (2002).

[45] A. V. Artamonov et al. (BNL-E949 Collaboration), Phys. Rev. D 79, 092004 (2009).

[46] E. C. Gil et al. (NA62 Collaboration), J. High Energy Phys. 05 (2019) 182. 
[47] D. Banerjee et al. (NA64 Collaboration), Phys. Rev. Lett. 118, 011802 (2017).

[48] D. Banerjee et al. (NA64 Collaboration), Phys. Rev. D 97, 072002 (2018).

[49] D. Banerjee et al., Phys. Rev. Lett. 123, 121801 (2019).

[50] J. P. Lees et al. (BABAR Collaboration), Phys. Rev. Lett. 119, 131804 (2017).

[51] P. Ilten, Y. Soreq, M. Williams, and W. Xue, J. High Energy Phys. 06 (2018) 004.

[52] R. Essig, J. Mardon, M. Papucci, T. Volansky, and Y. M. Zhong, J. High Energy Phys. 11 (2013) 167.

[53] H. Davoudiasl, H. S. Lee, and W. J. Marciano, Phys. Rev. D 89, 095006 (2014).

[54] S. Adler et al. (E787 Collaboration), Phys. Rev. D 70, 037102 (2004).

[55] P. Fayet, Phys. Rev. D 74, 054034 (2006).

[56] P. Fayet, Phys. Rev. D 75, 115017 (2007).

[57] P. J. Fox, R. Harnik, J. Kopp, and Y. Tsai, Phys. Rev. D 84, 014028 (2011).

[58] H. Peng, Charm2018, Novosibirsk, Russia (2018), http:// indico.inp.nsk.su/event/10/session/1/contribution/68/ material/slides/0.pdf.

[59] A. E. Bondar et al. (Charm-Tau Factory Collaboration), Yad. Fiz. 76, 1132 (2013) [Phys. At. Nucl. 76, 1072 (2013)].

[60] S. h. Zhu, Phys. Rev. D 75, 115004 (2007).

[61] P.f. Yin, J. Liu, and S. h. Zhu, Phys. Lett. B 679, 362 (2009).

[62] Z. Liu and Y. Zhang, Phys. Rev. D 99, 015004 (2019).

[63] J. Liang, Z. Liu, Y. Ma, and Y. Zhang, arXiv:1909.06847.

[64] R. Essig, P. Schuster, and N. Toro, Phys. Rev. D 80, 015003 (2009).

[65] E. Ma and J. Okada, Phys. Rev. Lett. 41, 287 (1978); 41, 1759(E) (1978).

[66] K. J. F. Gaemers, R. Gastmans, and F. M. Renard, Phys. Rev. D 19, 1605 (1979).

[67] M. Ablikim et al. (BESIII Collaboration), Phys. Rev. D 96, 112008 (2017).
[68] C. Mana and M. Martinez, Nucl. Phys. B287, 601 (1987).

[69] S. Actis, P. Mastrolia, and G. Ossola, Phys. Lett. B 682, 419 (2010).

[70] T. Hahn, Comput. Phys. Commun. 140, 418 (2001).

[71] T. Hahn and M. Perez-Victoria, Comput. Phys. Commun. 118, 153 (1999).

[72] M. Ablikim et al. (BESIII Collaboration), Phys. Rev. D 83, 112005 (2011).

[73] Z. Liu, Y. H. Xu, and Y. Zhang, J. High Energy Phys. 06 (2019) 009.

[74] W.-D. Li, Y.-J. Mao, and Y.-F. Wang, International Journal of Modern Physics A (World Scientific, Singapore, 2009), pp. 9-21.

[75] Dayong Wang (private communication).

[76] http://english.ihep.cas.cn/bes/doc/2250.html.

[77] E. Kou et al. (Belle-II Collaboration), arXiv:1808.10567.

[78] H. Davoudiasl and W. J. Marciano, Phys. Rev. D 92, 035008 (2015).

[79] P. deNiverville, M. Pospelov, and A. Ritz, Phys. Rev. D 84, 075020 (2011).

[80] B. Batell, M. Pospelov, and A. Ritz, Phys. Rev. D 80, 095024 (2009).

[81] B. Batell, R. Essig, and Z. Surujon, Phys. Rev. Lett. 113, 171802 (2014).

[82] A. A. Aguilar-Arevalo et al. (MiniBooNE Collaboration), Phys. Rev. Lett. 118, 221803 (2017).

[83] E. Izaguirre, G. Krnjaic, P. Schuster, and N. Toro, Phys. Rev. D 91, 094026 (2015).

[84] E. Izaguirre, G. Krnjaic, P. Schuster, and N. Toro, Phys. Rev. Lett. 115, 251301 (2015).

[85] E. Izaguirre, Y. Kahn, G. Krnjaic, and M. Moschella, Phys. Rev. D 96, 055007 (2017).

[86] R. Essig, A. Manalaysay, J. Mardon, P. Sorensen, and T. Volansky, Phys. Rev. Lett. 109, 021301 (2012). 